\title{
Proton induced X-ray emission analysis of aberrant cowrie shells
}

\author{
Adele M. Luta \\ Florida Institute of Technology, Melbourne, Florida 32901 \\ Department of Physics, University of Florida, Gainesville, Florida 32611
}

\begin{abstract}
The Materials Analysis/Characterization and Ion Beam Reaction Group at the University of Florida operates a 1.7 million-volt Pelletron tandem accelerator with a Proton Induced X-ray Emission (PIXE) analysis facility. Dr. Chris Meyer from the Florida Museum of Natural History collaborated with this group to conduct an investigation to determine whether or not aberrant rostrate and melanistic cowrie shells have a surplus of heavy metals. Meyer's thesis stated this irregular growth and coloring of the shells was a result of the cowrie's biochemical pathway. The research group found no pattern that distinguished differences among the shells; therefore, no clear evidence could be concluded about the cowries' nature in this experiment. Meyer and the research group feel the confirmation of absence of heavy element abundance in rostrate and melanistic shells has led to a conclusive study at this time.
\end{abstract}

\subsection{Introduction}

The Materials Analysis/Characterization and Ion Bean Reaction Group at the University of Florida operates a 1.7 million-volt Pelletron Tandem accelerator. With a Proton Induced X-ray Emission (PIXE) system extension, the group conducts research involving collaboration among multiple departments at the university. One of these collaborations was with Dr. Chris Meyer from the Florida Museum of Natural History. Meyer contacted the group with the proposal to investigate whether or not aberrant rostrate and melanistic gastropod shells have a surplus of heavy metals.

The Model 5SDH Pelletron Tandem electrostatic accelerator was acquired from Los Alamos Scientific Laboratory at the end of 2000. The Pelletron design exceeds the performance of other accelerators with its endless inductively charged bead chain of alternating nylon and aluminum links. The accelerator has a 1.7 MV horizontal tandem system, which produces stable beams with currents up to 200nA. PIXE's major 
advantage over other techniques is its ability to measure very small absolute masses of an element [1]. Using AccuSpec and RobWin, analysis software, the X-ray spectrum produced can analyzed allowing for the detection of elements present in only a few parts per million.

The particular interest with the aberrant cowrie shells lies in the matrix structure of the rostrate and melanistic characteristics. Ore deposits composed of nickel, iron, cobalt, chromium, and copper were hypothesized to link the abnormalities found in the shells [2]. These shells dwell only in certain parts of the world where the ore deposits and coastal areas are in close proximity; one example is the coast off New Caledonia located in the South-West Pacific. The group will test regular shells as the control along with two different types of the abnormal ones. A confirmation of the increased amount in the cowries will add to the scientific community's understanding of the growth of these shells.

\subsection{Background}

\subsection{PIXE}

First introduced by the Lund Institute of Technology in 1970, PIXE gave a whole new dimension to elemental analysis. Unlike other spectroscopic techniques, PIXE is based on the physics of the atom not its chemistry. According to a website sponsored by Elemental Analysis Corporation, "PIXE is a technique which can be used for nondestructive, simultaneous elemental analysis of solid, liquid, thin film, and aerosol filter samples" [3]. With the use of a proton beam, as used in this experiment, two major advantages follow. First, there is a higher rate of data accumulation. Second, although it 
does still contain a range for best sensitivity $(20<Z<40)$, the overall sensitivity in lower elements is increased when compared to other processes [3].

PIXE is based on the physics of X-ray emission. As a result of the excitation to the atom by the incident protons, an electron is removed from the K, L, M, or N shells and an electron from the atom's outer orbit fills the vacancy. This set of events emits a soft x-ray characterized through its spectrum (peaks of K alpha, beta and L alpha, beta) [1]. A Si(Li) detector collects the X-rays. The signals from the detector are amplified and then analyzed by AccuSpec, a multi-channel analysis program. The Faraday cup at the end of the beam collects the charge and the current integrator records the total charge collected during the run. See fig. 1 for a simple PIXE setup.

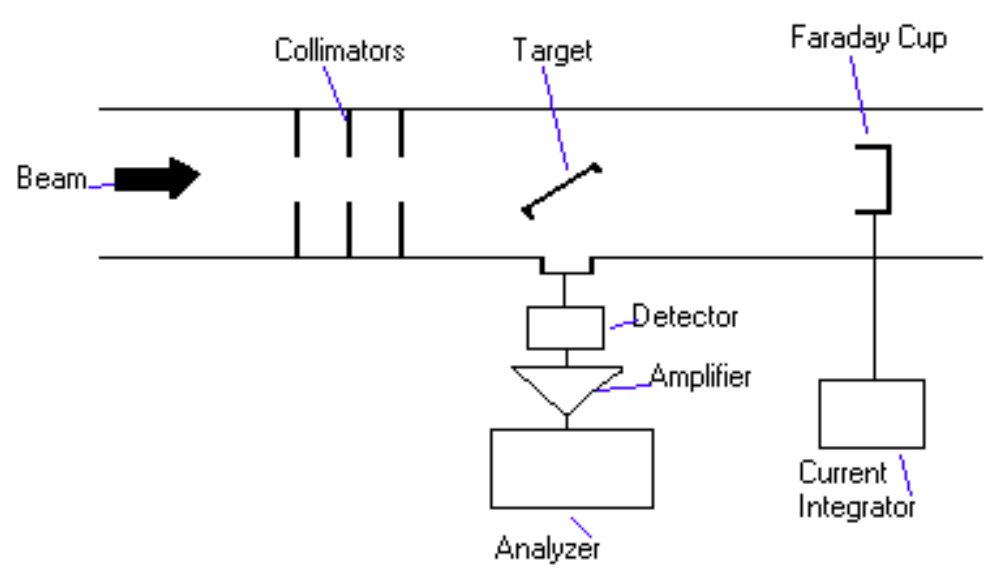

FIGURE 1: A simple outline of PIXE setup.

As an addition to the basic set-up, the researchers collected spectrum with two types of filters in place. Each filter is interchanged in front of the detector to decrease the bremsstrahlung and other low-energy radiation. A funny and regular filter gives a confirmation of each other's run. The "funny" filter contains a pinhole, which allows for $1 \%$ of the lower-energy radiation to get through compared to a regular filter (contains no hole). 


\subsection{Shells}

Since the mid 1800's, the irregular cowrie shells have drawn attention to New Caledonia. At first it was thought that these shells came from all over the island but later scientists later realized only a few coasts contained these highly valued shells. Unfortunately, this misdocumentation also led to the loss of the origins of many of the older samples particular origins on the island. Currently, scientists have drawn connections to the location of the rostrate and melanistic shells to particular coasts associated with large ore depositories from mining. The area is very specific (See Appendix A).

The cowries shells contain two defining characteristic which lead to their aberrant classification: rostration and melanism. Rostration is the excessive growth of the shell at its ends (See Figure 2). Melanism refers to an increase of pigment in the shell's upper layer (See Figure 3). In 1992, Meyer's master thesis proposed that a connection lie between the abnormalities in the cowries and the iron deposits through the animals' biochemical pathways [2]. The proposed activating elements included nickel, iron, cobalt, chromium, and copper. These elements lie within the highest sensitivity range of PIXE; therefore, the experiment is an excellent match for the lab.

\subsection{Experiment}

After obtaining the museum's permission to break the rare and monetarily valuable shells, Meyer carefully picked one shell from each category which he felt would give well: defined results. See Table I below for the significant characteristic of each shell. Meyer recorded digital photographs of each shell. See Appendix B for digital pictures. Next, he broke the shells in half making sure to watch for the cleanest layer 

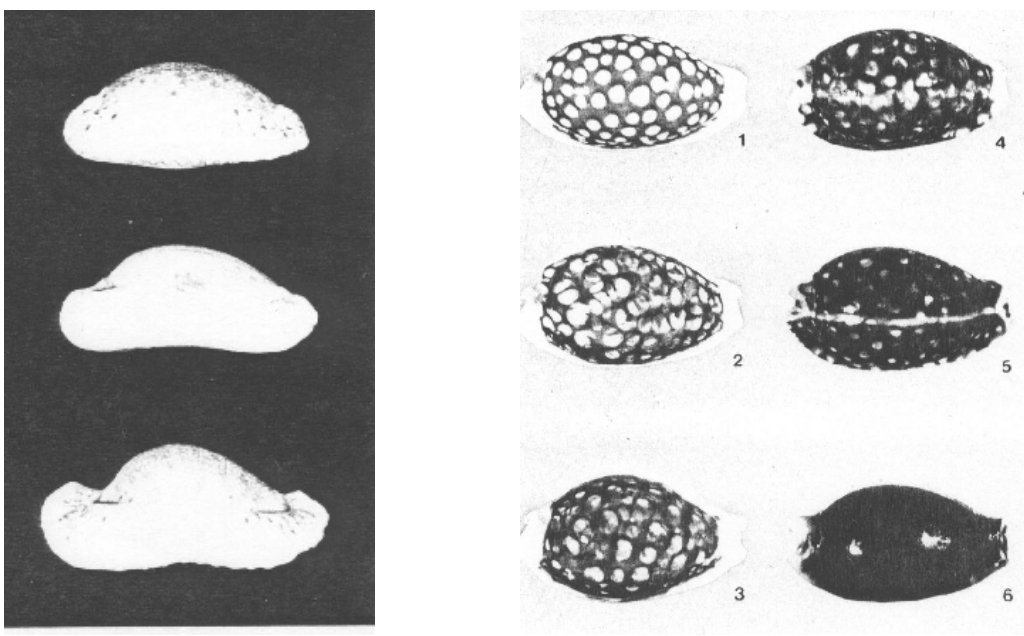

FIGURE 2 shows an increase from top to bottom shells with rostration. [5] FIGURE 3 displays an increase from top to bottom cowrie shells with melanistic characteristics. [5]

separation. Finally, he placed the ends and top few layers from each shell in plastic containers. At all times, Meyer eliminated chances of contamination by keeping the shells away from any metallic surfaces.

TABLE I. Description of experimental cowries.

\begin{tabular}{|l|c|c|}
\hline \multicolumn{1}{|c|}{ Type\& Position } & Felina Shell & Arabica Shell \\
\hline Melanistic (single top) dorsal prismatic & & $X$ \\
\hline Melanistic dorsal top layer & $X$ & \\
\hline Regular dorsal top layer & $X$ & $X$ \\
\hline Rostrate anterior end & $X$ & \\
\hline Regular anterior end & $X$ & \\
\hline
\end{tabular}

After receiving the shells, the researcher crushed each small piece of shell separately by hand utilizing a mortar and pestle. Made of powdered synthetic sapphire this particular mortar and pestle allowed the researcher to break the shell down to a very fine powder. Next, the researcher placed a few milligrams of the shell on an aluminized Mylar thin film. Using expediency the researcher dropped $50 \mu 1$ of a $1 \%$ polystyrene solution in benzene on the target and proceeded to smooth out the glue and shell powder before the glue dried. Once again, to eliminate any further contamination, only glass or Teflon coated instruments were employed. 
To obtain optimal data, the research group ran each target four times, twice with the funny filter and twice with the regular filter. The average total proton counts recorded on one data run is 40 micro coulombs. Due to the large amount of $\mathrm{Ca}$ in the shells the research group decreased the beam intensity to around $20 \mathrm{nA}$. However, this recorded intensity varied during the run and the records only display the end reading. The average intensity resided at $20 \mathrm{nA}$.

\subsection{Data Analysis}

In order to ensure an accurate reading of the spectra, the researchers perform two checks. The first is the analyzing of the data using RobWin. The researcher matches only two peaks for RobWin, and the software calculates all other aspects of the spectrum. After a few minutes, RobWin accounts for the background radiation, identifies small peaks not easily seen by the naked eye, and separates any overlapping peaks. One of the most useful outputs of RobWin is its ability to report the number of counts of a particular element. However there are a few phenomena which RobWin has a hard time identifying. Double peaks are one example. Double peaks or pulse pile-ups occur when a high concentration of $x$-rays arrive at the detector at the same time. The detector records two identical pulses as a mirror pulse at double the energy value. Therefore, the researchers also check each spectrum visually.

With the confirmation of the peaks, the researchers now take steps to confirm the number output. The research group uses $\mathrm{Ca}$ as an internal standard. With the absence of a control inputted standard, the numerical data may only be compared in ratios. The researchers evaluate the experimental ratios with standard ratios. Orchard leaves, pine needles, and spinach give the most optimal elemental matches allowing for the 
comparison between the different shells. See Appendix $C$ for the equations used in the ratio calculations. Finally, in order make accurate scientific conclusions, the researchers meet with Meyer to combine the PIXE data and biological theory.

\subsection{Results}

\subsection{PIXE Run Comparison}

The regular filter, combined with around 800 counts per second average, displays a lower double peak than the funny filter. Analyzing to include Al, S, and P when running RobWin only increases the program calculation time and does not affect the heavy element analysis. An average run of $40 \mu \mathrm{C}$ gives the cleanest spectrum. See Appendices D and E for two sample spectra.

\subsection{Type and Part of Shell Comparison}

After running appropriate calculations to compare variable element/calcium ratios, the researchers fine that the regular and rostrate and/or melanistic cowries produced the same results for both filters. See Tables II and III below for a detailed ratio account for each filter and shell.

TABLE II. Element/ca comparison for Arabica elements

\begin{tabular}{|l|l|l|l|l|}
\hline Element & Funny Filter & Regular Filter & Funny & Regular \\
\hline $\mathrm{Cr}$ & 0.0044 & 0.13 & 0.338 & 1.088 \\
\hline $\mathrm{Cu}$ & 00.60 & 0.165 & 0.042 & 0.076 \\
\hline $\mathrm{Fe}$ & 0.14 & 0.22 & 1.22 & 2.22 \\
\hline $\mathrm{K}$ & 170 & 1860 & 180 & 1640 \\
\hline $\mathrm{Sr}$ & 83 & 176 & 52 & 108 \\
\hline $\mathrm{Zn}$ & 0.74 & 0.97 & 0.41 & 0.38 \\
\hline
\end{tabular}

Melanistic or Regular cowrie; Numbers are X $10^{-4}$ 
TABLE III. Element/ca comparison for Felina elements

\begin{tabular}{|l|l|l|l|l|l|l|l|l|}
\hline Element & $\begin{array}{l}\text { Dorsal } \\
\text { Top } \\
\text { Funny }\end{array}$ & $\begin{array}{l}\text { Dorsal } \\
\text { Top } \\
\text { Regular }\end{array}$ & $\begin{array}{l}\text { Anterior } \\
\text { End } \\
\text { Funny }\end{array}$ & $\begin{array}{l}\text { Anterior } \\
\text { End } \\
\text { Regular }\end{array}$ & $\begin{array}{l}\text { Dorsal } \\
\text { Top } \\
\text { Funny }\end{array}$ & $\begin{array}{l}\text { Dorsal } \\
\text { Top } \\
\text { Regular }\end{array}$ & $\begin{array}{l}\text { Anterior } \\
\text { End } \\
\text { Funny }\end{array}$ & $\begin{array}{l}\text { Anterior } \\
\text { End } \\
\text { Regular }\end{array}$ \\
\hline $\mathrm{Cr}$ & 0.34 & 0.08 & 0.12 & 0.49 & 0.07 & 0.23 & 0.16 & 0.45 \\
\hline $\mathrm{Cu}$ & 0.098 & 0.045 & 0.037 & 0.067 & 0.040 & 0.069 & 0.056 & 0.078 \\
\hline $\mathrm{Fe}$ & 1.3 & 0.8 & 1.2 & 2.1 & 0.6 & 1.1 & 1.0 & 1.8 \\
\hline $\mathrm{K}$ & 180 & 1810 & 170 & 1920 & 180 & 1980 & 180 & 1700 \\
\hline $\mathrm{Sr}$ & 150 & 70 & 65 & 132 & 47 & 103 & 50 & 106 \\
\hline $\mathrm{Zn}$ & 0.16 & 0.19 & 0.17 & 0.15 & 0.51 & 0.41 & 0.13 & 0.30 \\
\hline
\end{tabular}

Melanistic or Regular cowrie; Numbers are X $10^{-4}$

\subsection{Conclusions}

\subsection{PIXE Run}

The results from the difference in running the funny filter to the regular filter upheld the researchers' initial projected limitations.

\subsection{Type and Part of Shell}

Any ratio variation is due to the variation in the calcium contained in the shell, which acted as an internal standard. The replicated bias consistency toward the regular and rostrate and/or melanistic cowries indicates a reliable data set. Meyer's added expertise informed the research group any ratio less than a factor of two would not necessarily indicate a difference in all shells due to the well-known law in nature ${ }^{1}$. This leaves only the difference in $\mathrm{Fe}$ and $\mathrm{Cr}$ is distinct in the differences between the two types of shells. The Fe concentration is 10 times higher in the regular Arabica vs. the melanistic Arabica $\left(2.2 \times 10^{-5}\right.$ melanistic, $22.2 \times 10^{-5}$ regular). The $\mathrm{Cr}$ follows the same bias towards the regular with an 8.5 multiple increase $\left(13.0 \times 10^{-6}\right.$ melantistic, $108.8 \times 10^{-6}$ regular). Surprisingly, this increase in the regular shells indicates a contrary biological

\footnotetext{
${ }^{1} \mathrm{~A}$ common law in biology is that nature builds in a biological variation by a factor of two. For example, trees can withstand two times the wind force that they will ever see in their lifetime. In the case of the present experiment, unless an element is dramatically over double in element concentration, it cannot be counted as reliable difference.
} 
phenomenon than stated in Meyer's master thesis. No pattern stands out as a distinct difference between the shells; and therefore, no clear trace can be concluded about the cowries' nature in this experiment. In conclusion, the researchers disproved Meyer's master's thesis by showing no pattern of increase for heavy metal in the upper layers of the shells.

\subsection{Future Studies}

Based on the results of the experiment, Meyer concluded that due to the cowries' rarity and value it would be unnecessary to conduct any further runs. Both Meyer and the researchers felt confident in the data set. Meyer's only suggestion for further analysis would be to analyze the sponges, which are thought to be the food carrier of the heavy elements. However, these sponges do not survive in tanks and have a very large rate of decay when removed from the water. Therefore, until further scientific developments occur, the present experiment has provided the best possible data regarding whether or not aberrant rostrate and melanistic cowrie shells contain a surplus of heavy metals.

\section{Acknowledgments}

Much appreciation goes to the National Science Foundation (NSF) for sponsoring the REU program. Thanks to Drs. Ivan Kravchenko, Henri Van Rinsvelt, and F.E. Dunnam for their guidance and patience. Thanks to Dr. Chris Meyer for taking the time to explain biology to a physics student. A large thank you goes to Ralph Kelly and John Kuharik for their laughter and teamwork. Thanks goes to Drs. Kevin Ingersent and Alan Dorsey for all of their organization efforts to make this summer as beneficial as possible. Thank you to all of the guest speakers who presented techniques in scientific communication. Finally, thanks to Katie Slinkard for assistance in the long calculations. 


\section{References}

[1] Waldemar H. Scharf, Particle Accelerators-Applications in Technology and Research (John Wiley \& Sons Inc., New York, 1989).

[2] Christopher Porter Meyer, Pattern Formation in Cypraeid Gastropds: A Specific Case of Pattern Formation Leading to a General Model (University of California, Berkeley, Berkeley, California, 1992).

[3] Elemental Analysis Corporation, http://www.elementanalysis.com/PixeService.htm $(7 / 10 / 01)$.

[4] Sasha dos-Santos, Ivan Kravchenko, and F.E. Dunnam, Detection of Trace Elements in Meteorites Using PIXE (University of Florida, Gainesville, Florida, 2000).

[5]J. M. Chatenay, Niger and Rostrate Cowries of New Caledonia (Inprimeries Reunies de Nournea, Nournea, New Caledonia, 1977). 


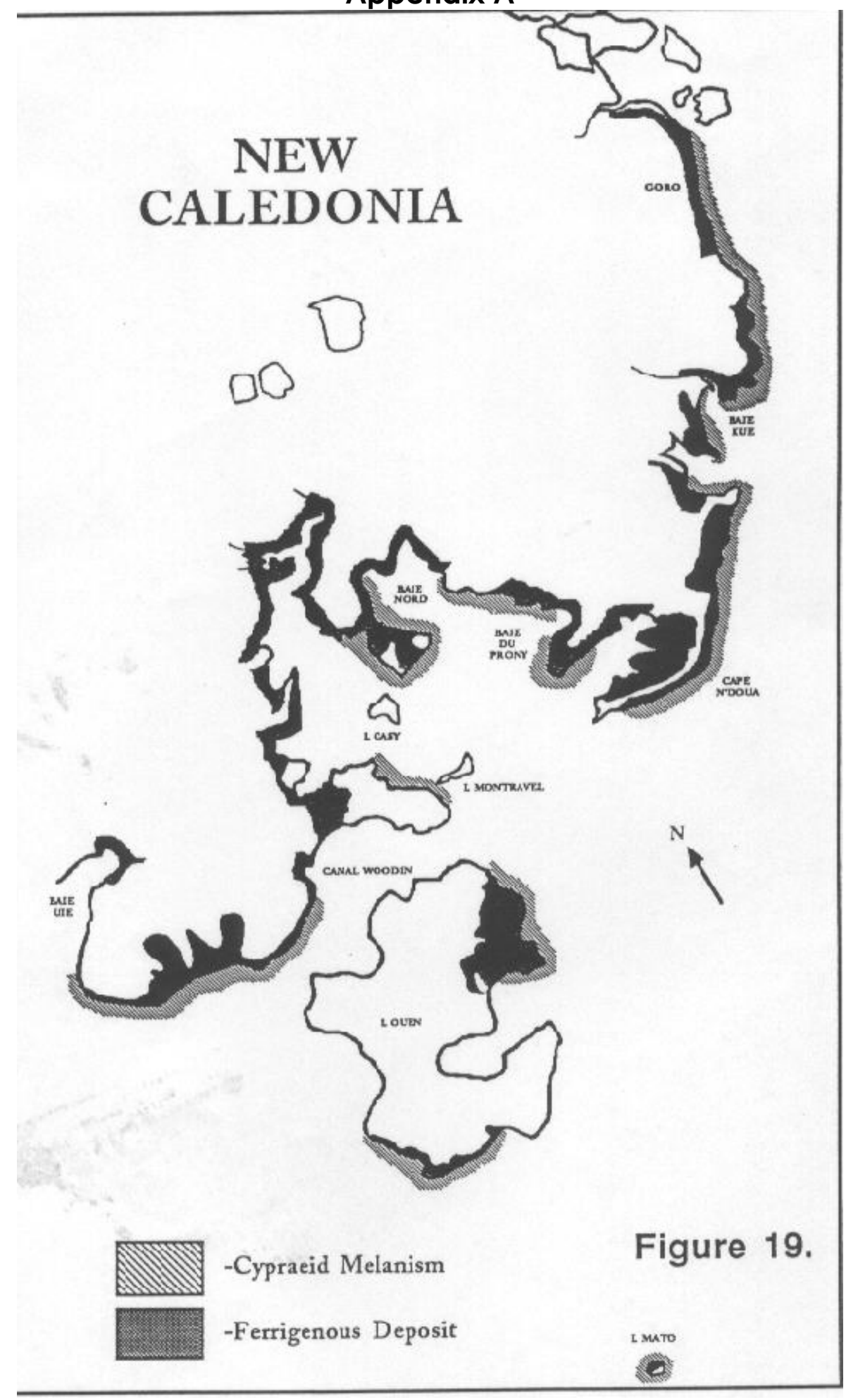

Map of New Caledonia located off the coast of Australia [5]. 


\section{Appendix B}

Arabica Regular

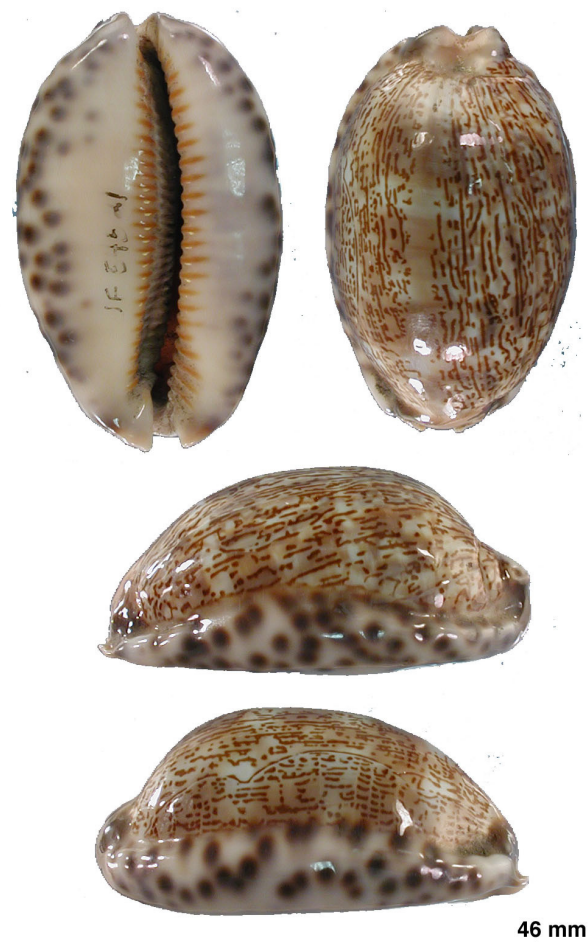

Felina Melanistic and Rostrate

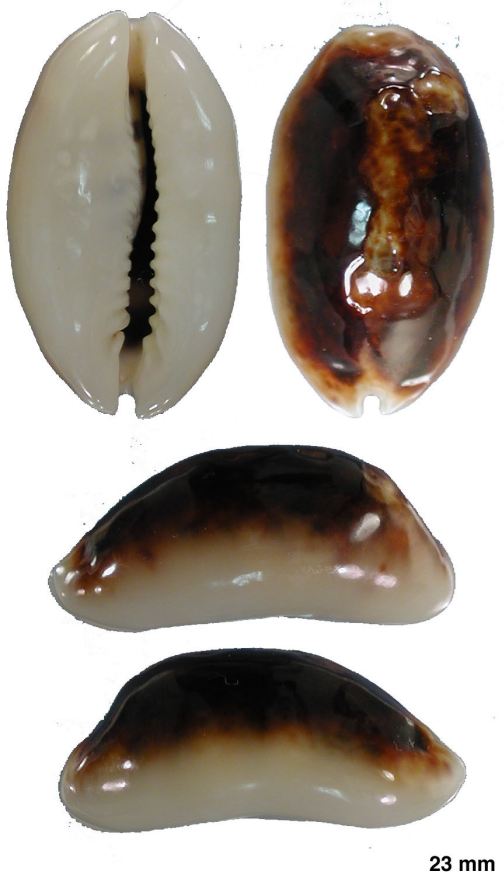

Arabica Melanistic

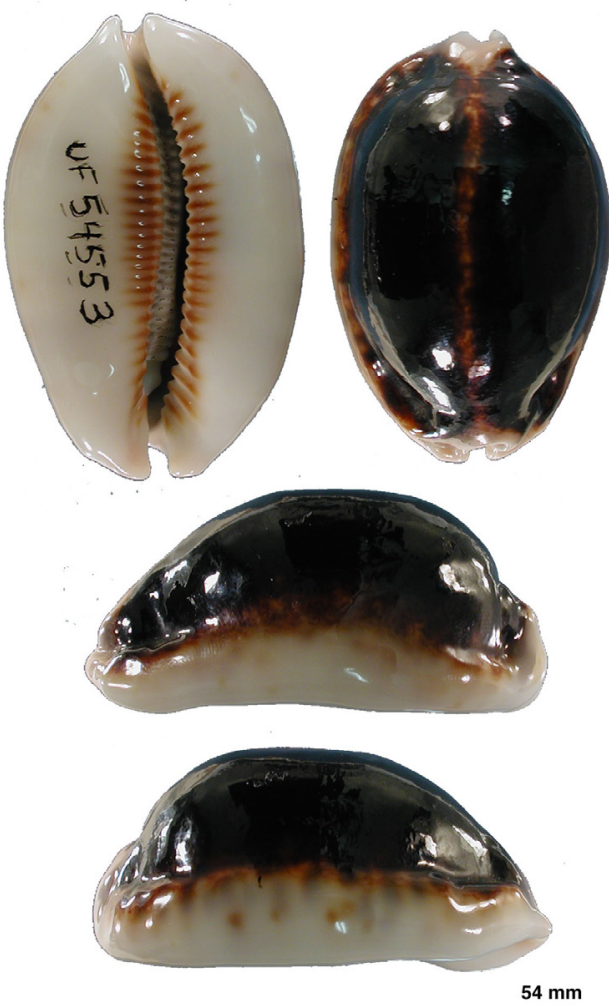

Felina Regular

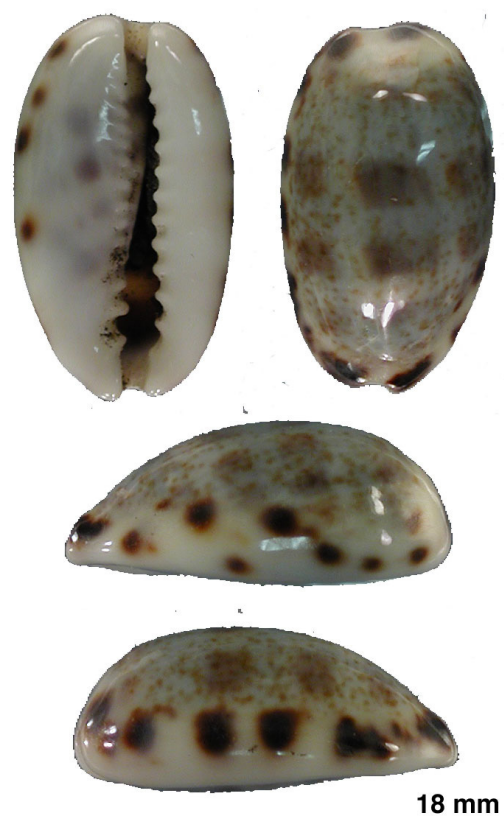




\section{Appendix C}

Sample calculation for one run of iron $(\mathrm{Fe})$ with one kind of filter

\section{Counts Fe \\ Counts $\mathrm{Ca}=\mathrm{A}$}

\section{Parts per million $\mathrm{Fe}$}

Parts per million $\mathrm{Ca}=\mathrm{B}$

$$
\underline{\mathrm{A}}=\mathrm{C}
$$

$\sum \mathrm{C}$

Number of Standards Used $=\mathrm{C}_{\text {avg }}$

Counts $\mathrm{Fe}_{\text {shell }}$

Counts $\mathrm{Ca}_{\text {shell }}=\mathrm{A}_{\mathrm{s}}$

$$
\frac{\mathrm{A}_{\mathrm{s}}}{\text { Cavg }}=\text { Melanistic ratio }
$$

Melantistic Ratio

Regular Ratio = Final Ration for Comparison 


\section{Appendix D}

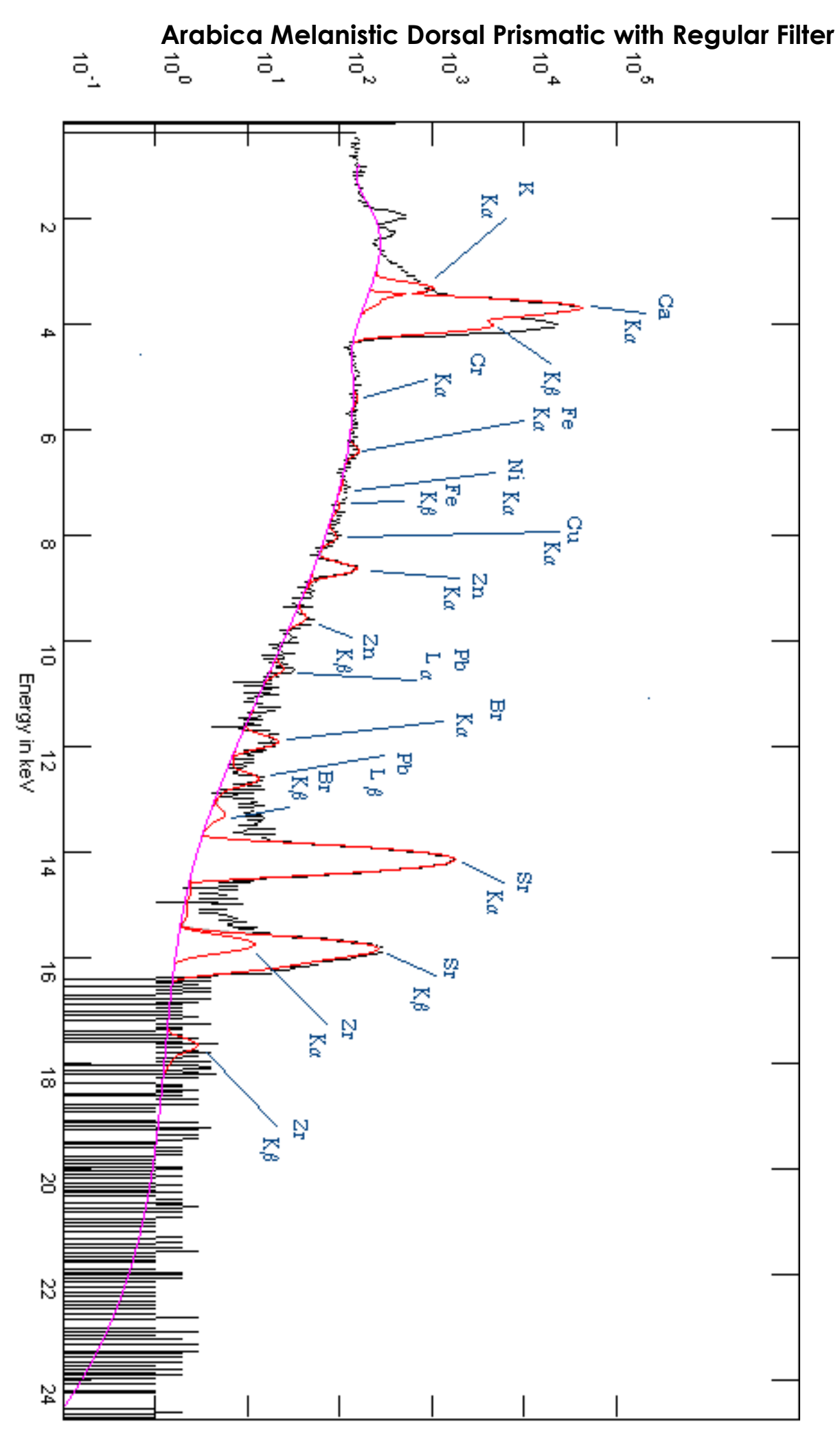




\section{Appendix E}

Arabica Regular Dorsal Top Layer with Regular Filter

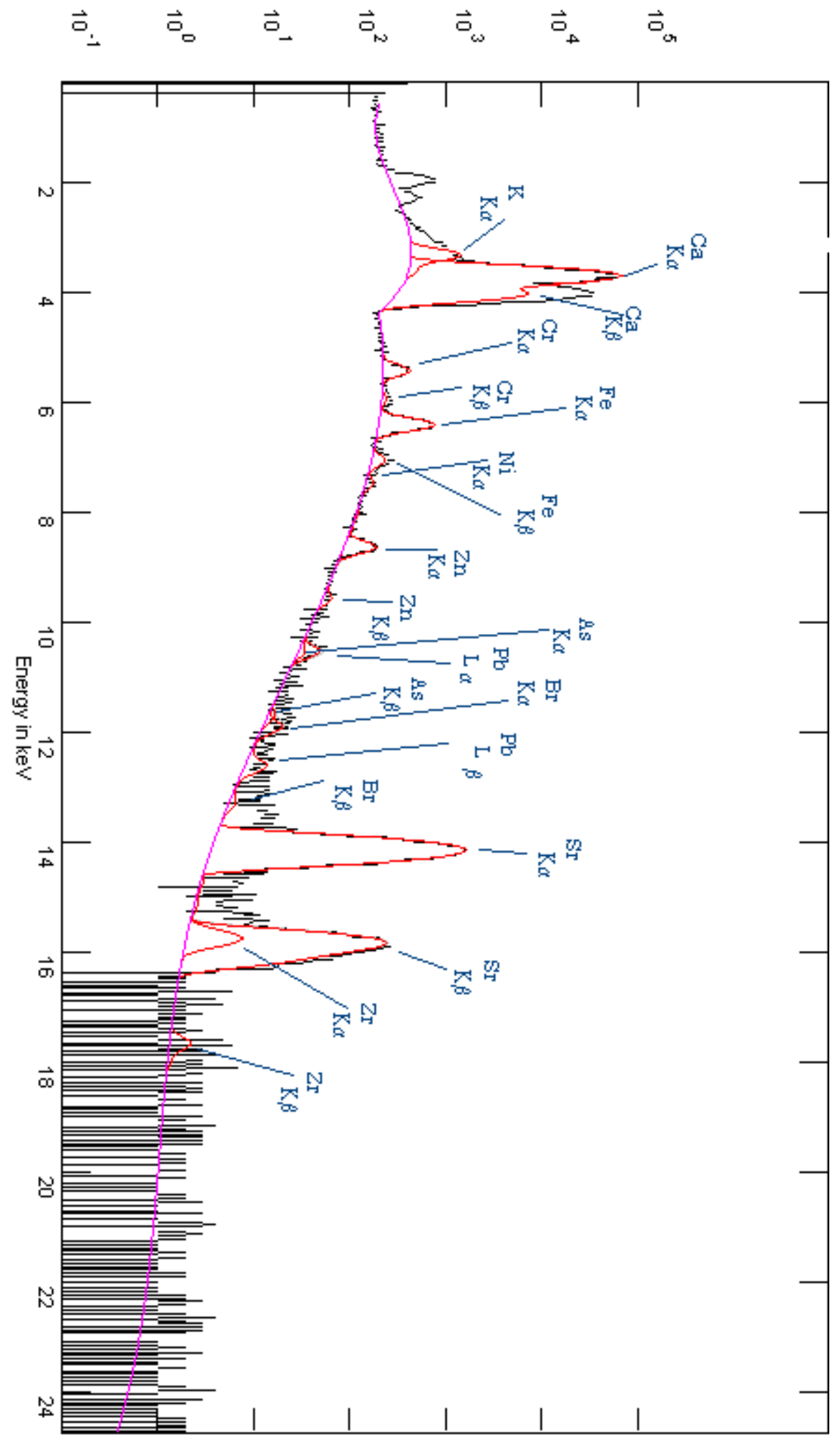

